\section{Expression of the metastasis suppressor gene KISS1 in uveal melanoma}

CMO Martins, BF Fernandes, E Antecka, S Di Cesare, JJC Mansure, J-C Marshall and MN Burnier Jr

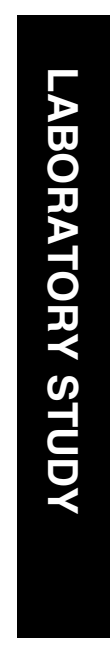

Department of Ophthalmology, Henry C Witelson Ocular Pathology Laboratory, McGill University Health Centre, Montreal, Quebec, Canada

Correspondence: CMO Martins, Henry C Witelson Ocular Pathology Laboratory, McGill University Health Centre,

3775 University Room 216, Montreal, Quebec,

Canada H3A 2B4

Tel: + 5143987192

ext: 00384.

Fax: + 5143985728

E-mail: claudia.martins@ mail.mcgill.ca

Received: 26 July 2007 Accepted in revised form: 17 December 2007 Published online: 25 January 2008

Advances in the treatment of UM failed to improve survival rates in the last decades. Targets to treat metastasis are desperately needed.

Metastasis Suppressor Gene KISS1 is characterized in UM for the first time and its relationship with survival rate of UM patients strongly suggests a possible role for KISS1 as a prognostic marker and a therapeutic target.

This work was previously presented as a poster at ARVO 2007.
Eye (2008) 22, 707-711; doi:10.1038/sj.eye.6703090; published online 25 January 2008

\section{spontaneous, macroscopic metastases without} affecting the growth rate of the primary tumour. ${ }^{8}$ 
The KISS1 gene has been reported to be a metastasis suppressor gene in human skin melanoma and breast cancer cells. ${ }^{8}$ Nash et al were the first to show that the introduction of KISS1 into highly metastatic human melanoma cell lines C8161 and MelJuSo suppressed in vivo metastase to the lung by more than $95 \%$.9,10 Interestingly, introduction of KISS1 into a metastatic breast cancer cell line MDA-MB-435 also showed a $>95 \%$ suppression of metastases to the lung. ${ }^{11}$ It was also demonstrated that loss of KISS1 mRNA expression correlates with conversion from the benign to the malignant phenotype of cutaneous human melanoma. ${ }^{12}$ These data strongly suggest that KISS1-mediated metastasis suppression might be pertinent in different tumours, a conclusion that surfaced in subsequent studies. ${ }^{13-18}$ Moreover, reduced KISS1 expression became a strong prognostic marker in patients with urinary bladder cancer ${ }^{14}$ and gastric carcinoma. ${ }^{16}$ In general, loss or reduction of KISS1 expression in several different tumour types negatively affects survival. ${ }^{19}$

KISS1 encodes a 145 amino-acid residue peptide that is further processed. One of the products, a 54 amino-acid peptide, is called Metastin or Kisspeptin-54 and is a natural ligand to a G-coupled receptor known as GPR $54 .^{20}$ Its mechanisms of action remain elusive but experimental evidence shows that KISS1 secretion is required for multiple organ metastasis suppression and for maintenance of disseminated cells in a dormant state. $^{21}$

Despite the obvious clinical importance of cancer metastasis, the process remains incompletely characterized at the molecular level. Therefore, a better understanding of these processes is essential to develop novel and specific drugs to prevent and treat UM metastasis. However, to date, characterization of KISS1 expression in melanoma has only been undertaken in skin melanoma ${ }^{19}$ and remains to be investigated in UM.

This study aims to evaluate the immunohistochemical expression of KISS1 in UM cell lines with different metastatic potential. In addition, characterization of the immunohistochemical expression in tissues of patients with primary UM and its possible association with survival are assessed.

\section{Materials and methods}

\section{Immunocytochemistry}

Cytospins of the five human UM cell lines with different metastatic potentials (92.1, SP6.5, OCM-1, UW-1, and MKT-BR) were made using a Cytospin3 machine (Shandon Scientific Limited, Astmoor, UK). Cells from culture were diluted to a concentration of 250000 cells per $\mathrm{ml}$, and a $300 \mu \mathrm{l}$ solution at that concentration was used for each spin to be evenly plated on each slide. All slides were then immunostained with primary antihuman monoclonal antibody against KISS1 (M05, Abnova Corporation, Heildelberg, Germany) using the Ventana Benchmark LT fully automated machine (Ventana Medical Systems Inc., Tucson, AZ, USA) programmed to use a standard avidin-biotin complex method.

The UM cell lines 92.1, SP6.5, and MKT-BR were established by Dr Jager (University Hospital Leiden, The Netherlands), Dr Pelletier (Laval University, Quebec, Canada), and Dr Belkhou (CJF INSERM, France), respectively. Dr Albert (University of WisconsinMadison, USA) established the OCM-1 and UW-1 cell lines. ${ }^{22,23}$

\section{RNA extraction}

Total cellular RNA was extracted from the five human UM cell lines using the Qiagen RNeasy kit (Qiagen, Mississauga, Ontario, Canada) as per the manufacturer's recommendations. Briefly, cells were disrupted and homogenized using the included lysate buffer and ground with a $20 \mathrm{G}$ (half) syringe, as per the manufacturer's instructions. The lysate was then centrifuged to remove any insoluble material. One volume of $70 \%$ ethanol was added to the lysate and mixed before the solution was added to the included RNeasy mini column. Following centrifuging the columns were then washed twice using the included buffer solutions. Total cellular RNA was then eluted using RNase-free water.

Expression levels of KISS1 mRNA and GPR54 mRNA were then determined by real time-PCR using QuantiTect one-step SYBR Green PCR method (Qiagen) as per the manufacturer's instructions. A Chromo4 thermocycler (MJ Research, Waltham, MA, USA) was used for all experiments and all results were analysed using the GeneEx software. QuantiTect primer assay pairs (Qiagen) for KISS1 and GPR54 were used. Beta-actin levels were assessed in all experiments for the purposes of normalization.

\section{Patients}

Thirty-seven patients diagnosed with choroidal melanoma at the Henry C Withelson Laboratory, Royal Victoria Hospital, McGill Health Cancer Centre were studied. Their medical charts and Cancer registry entries were reviewed to provide information on the development of distant metastasis.

UM specimens obtained by enucleation were formalinfixed, paraffin-embedded, and each specimen contained sufficient material for hematoxylin and eosin and 
immunohistochemistry. Tumours presenting extensive necrosis that precluded an appropriate evaluation of histopathological features were excluded.

\section{Immunohistochemistry}

Protein expression was performed using the monoclonal anti-KISS1 antibody, in the Ventana Benchmark LT fully automated machine. The pathway KISS1 staining module was used according to the protocol and instructions provided by Ventana Medical System Inc.

The fully automated process of bar coding labelled slides including baking of the slides, solvent-free deparaffinization, and cell conditioning 1 (Tris-EDTA buffer $\mathrm{pH}$ 8.0) antigen retrieval for $30 \mathrm{~min}$. Slides were incubated with the mouse monoclonal antibody against KISS1 at a dilution of $1: 50$ for $30 \mathrm{~min}$ at $37^{\circ} \mathrm{C}$, followed by application of biotinylated secondary antibody $(8 \mathrm{~min}$, $\left.37^{\circ} \mathrm{C}\right)$, then an avidin-streptavidin enzyme conjugate complex $\left(8 \mathrm{~min}, 37^{\circ} \mathrm{C}\right)$. Finally the antibody was detected by Fast Red chromogenic substrate and counterstained with hematoxylin. As a positive control, sections of placenta were used, and for negative controls the primary antibody was omitted.

The immunostaining was evaluated in a semiquantitative method based on the percentage of viable cells showing positive expression $(0=$ no staining;
$1=$ staining of $<40 \%$ of the tumour; $2=$ staining of $>40 \%$ of the tumour) and intensity ( $0=$ no staining; $1=$ weak staining; $2=$ moderate staining; $3=$ strong staining). Two pathologists, who were unaware of the follow-up of each patient, performed the analysis. Total score was obtained by the sum of extent and intensity and finally organized: group $0=0-1-2$ and group $1=3-4$.

\section{Statistical analysis}

The incidence of metastasis was assessed using the Kaplan-Meier survival analysis test and log-rank test. A $P$-value of less than 0.05 was considered to be statistically significant. Calculations were computer-based (SPSS 11.5; SPSS Inc., Chicago, IL, USA). All data accumulation was in accordance with country and provincial laws, and the tenets of the Declaration of Helsinki.

\section{Results}

Staining of the human UM cell lines was cytoplasmatic in all five cell lines. The staining intensity was ranked as equivalents in all cell lines, with exception of UW-1, the least aggressive of the cell lines (Figure 1a), which had the strongest immunoexpression.
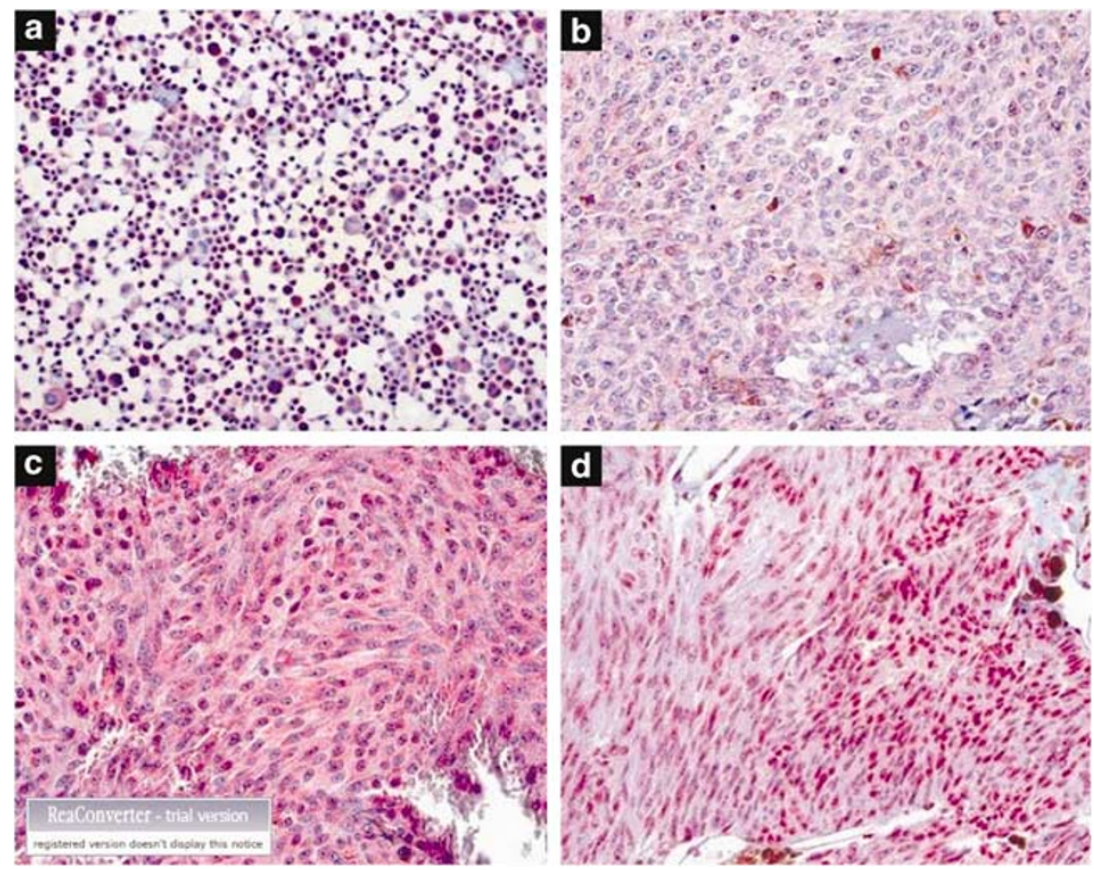

Figure 1 (a) Cytospin of UW-1 showed the strongest immunoexpression of KISS1 (original magnification $\times 400$ ). (b) Weak expression of KISS1 in an epithelioid-cell type uveal melanoma (UM) (original magnification $\times 400$ ). (c) Strong expression of KISS1 in a spindle cell type UM (original magnification $\times 400$ ). (d) Spindle cell type UM displaying nuclear staining for KISS1 (original magnification $\times 400$ ). 


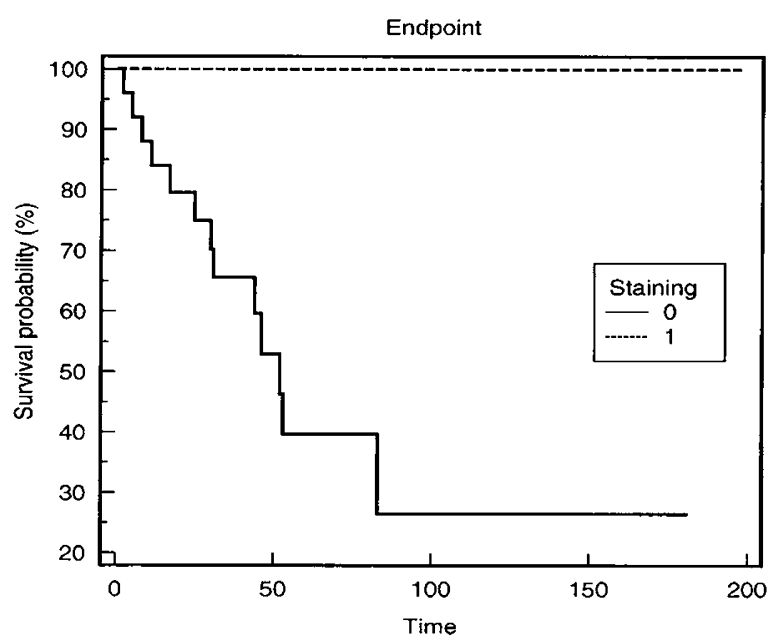

Figure 2 Kaplan-Meier test of uveal melanoma patients $(P<0.0001)$. Group 0 (low immunostaining of KISS1) and group 1 (high immunostaining of KISS1).

Real-time PCR analysis revealed that KISS1 and GPR54 were expressed in all five cell lines.

Eighty-four percent of the paraffin-embedded slides were positive for KISS1 ( 31 of 37 slides). All positive specimens had cytoplasmatic staining with only one exception, which had nuclear staining (Figure $1 \mathrm{~b}-\mathrm{d}$ ).

In the high staining group, no cases of metastasis were found (12 specimens). Conversely, in the low staining group, 13 cases of metastasis were observed in a total of 25 cases. There was a statistically significant association between the immunoexpression levels of KISS1 and survival rate of UM patients. (Kaplan-Meier; log-rank test $P<0.0001$ ) (Figure 2).

Regarding non-neoplastic ocular tissues, KISS1 stained normal optic nerve and all layers of the retina. The nonpigmented epithelium of the ciliary body was also positive for KISS1. The muscular fibres of the ciliary body showed a positive reaction, albeit with less intensity. No immunostaining of any other ocular structure was seen.

\section{Discussion}

To the best of our knowledge, this is the first time that the MSG KISS1 has been identified in UM, since all previous melanoma studies regarding this gene have been focused on skin melanoma. Owing to the significance of the metastatic process, identification of molecular targets and their mechanisms of action are essential. Therefore, targets that can possibly prevent the proliferation of metastases once they have already disseminated will be most advantageous in the treatment of patients with metastatic disease.

MSGs suppress the formation of metastasis, as they act in different pathways and sites. ${ }^{24}$ KISS1 and MKK4 are genes thought to be involved in the dormancy phase. ${ }^{7}$
KISS1 should be included in the list of targets that can maintain cells in a dormant state and have an immediate impact on patient survival. It is potentially of even further interest due to the fact that it encodes a secreted peptide, which interacts with a G-coupled receptor.

Our study demonstrated that KISS1 is expressed in human UM cell lines as well as UM human tissue. Measurements of the mRNA of KISS1 and its receptor GPR54 were performed in all five human UM cell lines and showed the presence of both of these genes, validating the immunocytochemical results.

Of significant interest was the strong correlation between patient survival rate and immunoexpression levels of KISS1 (Figure 2). KISS1 immunoreactivity was significantly associated with a lower risk of metastatic disease. However, further studies should be considered with larger sample sizes to assess the reproducibility of KISS1 expression in UM and its role as a prognostic factor.

Some structures of the normal eye showed positive immunostaining, including the optic nerve and retina. This might be due to the fact that the product of the KISS1 gene is secreted and processed to produce polypeptides, termed kisspeptins. Careful examination of the sequence revealed that KISS1 has characteristics in common with neuropeptides, which include a secretion signal, several dibasic cleavage sites and a cleavage amidation site. ${ }^{19}$ Structures derived from the neuronal system, such as the optic nerve and retina, may present cross reactivity.

The mechanism of action of KISS1 is still unknown. ${ }^{19}$ Experimental evidence to date suggests that KISS1 may suppress metastatic proliferation at the secondary site in several ways. One of the mechanisms suggests that secreted kisspeptins, or stromal peptides induced by kisspeptins, may deposit in the extracellular matrix altering its organization, structure, or composition in such a way that it provides an antiproliferative signal to adjacent metastatic cells. ${ }^{19}$ This mechanism may, in part, be explained by the way KISS1 interacts with metalloproteinases (MMPs).

MMP-9 expression has been shown to be upregulated in tumour cells and is correlated with invasion and metastasis. ${ }^{25,26}$ Regarding UM, MMP9 was associated with significantly worse prognosis. ${ }^{27}$ KISS1 is able to diminish MMP9 expression by reducing nuclear factor$\kappa \mathrm{B}$ binding to its promoter, ${ }^{28}$ and it has been suggested that MMPs may serve as negative regulators of KISS1. ${ }^{29}$ Our findings that demonstrate an inverse relationship between KISS1 expression and prognosis support the concept that KISS1 and MMP9 may somehow be related.

In conclusion, there is a need for new prognostic factors in UM and for new molecular targets concerning metastasis. Since downregulation of KISS1 seems to play 
a role in the dormancy phase of the metastatic cascade, further studies are needed to prove KISS1 as a probable prognostic factor in UM and as a potential therapeutic target.

\section{Acknowledgements}

This research was supported by the Cedars Cancer Institute of the Mcgill University Health Centre, Canada.

\section{References}

1 Singh AD, Bergman L, Seregard S. Uveal melanoma: epidemiologic aspects. Ophthalmol Clin North Am 2005; 18: 75-84, viii.

2 Kujala E, Makitie T, Kivela T. Very long-term prognosis of patients with malignant uveal melanoma. Invest Ophthalmol Vis Sci 2003; 44: 4651-4659.

3 Singh AD, Borden EC. Metastatic uveal melanoma. Ophthalmol Clin North Am 2005; 18: 143-150, ix.

4 Palmieri D, Horak CE, Lee JH, Halverson DO, Steeg PS. Translational approaches using metastasis suppressor genes. J Bioenerg Biomembr 2006; 38: 151-161.

5 Demicheli R, Abbattista A, Miceli R, Valagussa P, Bonadonna $G$. Time distribution of the recurrence risk for breast cancer patients undergoing mastectomy: further support about the concept of tumor dormancy. Breast Cancer Res Treat 1996; 41: 177-185.

6 Crowley NJ, Seigler HF. Relationship between disease-free interval and survival in patients with recurrent melanoma. Arch Surg 1992; 127: 1303-1308.

7 Steeg PS. Tumor metastasis: mechanistic insights and clinical challenges. Nat Med 2006; 12: 895-904.

8 Yoshida BA, Sokoloff MM, Welch DR, Rinker-Schaeffer CW. Metastasis-suppressor genes: a review and perspective on an emerging field. J Natl Cancer Inst 2000; 92: 1717-1730.

9 Miele ME, Robertson G, Lee JH, Coleman A, McGary CT, Fisher $\mathrm{PB}$ et al. Metastasis suppressed, but tumorigenicity and local invasiveness unaffected, in the human melanoma cell line MelJuSo after introduction of human chromosomes 1 or 6. Mol Carcinog 1996; 15: 284-299.

10 Welch DR, Chen P, Miele ME, McGary CT, Bower JM, Stanbridge EJ et al. Microcell-mediated transfer of chromosome 6 into metastatic human C 8161 melanoma cells suppresses metastasis but does not inhibit tumorigenicity. Oncogene 1994; 9: 255-262.

11 Lee JH, Welch DR. Suppression of metastasis in human breast carcinoma MDA-MB-435 cells after transfection with the metastasis suppressor gene, KiSS-1. Cancer Res 1997; 57: 2384-2387.

12 Shirasaki F, Takata M, Hatta N, Takehara K. Loss of expression of the metastasis suppressor gene KiSS1 during melanoma progression and its association with $\mathrm{LOH}$ of chromosome 6q16.3-q23. Cancer Res 2001; 61: 7422-7425.

13 Ikeguchi M, Yamaguchi K, Kaibara N. Clinical significance of the loss of KiSS-1 and orphan G-protein-coupled receptor (hOT7T175) gene expression in esophageal squamous cell carcinoma. Clin Cancer Res 2004; 10: 1379-1383.
14 Sanchez-Carbayo M, Capodieci P, Cordon-Cardo C. Tumor suppressor role of KiSS-1 in bladder cancer: loss of KiSS-1 expression is associated with bladder cancer progression and clinical outcome. Am J Pathol 2003; 162: 609-617.

15 Stark AM, Tongers K, Maass N, Mehdorn HM, Held-Feindt J. Reduced metastasis-suppressor gene mRNA-expression in breast cancer brain metastases. J Cancer Res Clin Oncol 2005; 131: 191-198.

16 Dhar DK, Naora H, Kubota H, Maruyama R, Yoshimura H, Tonomoto $\mathrm{Y}$ et al. Downregulation of KiSS-1 expression is responsible for tumor invasion and worse prognosis in gastric carcinoma. Int J Cancer 2004; 111: 868-872.

17 Guan-Zhen Y, Ying C, Can-Rong N, Guo-Dong W, Jian-Xin $\mathrm{Q}$, Jie-Jun W. Reduced protein expression of metastasisrelated genes (nm23, KISS1, KAI1 and p53) in lymph node and liver metastases of gastric cancer. Int J Exp Pathol 2007; 88: $175-183$.

18 Jiang Y, Berk M, Singh LS, Tan H, Yin L, Powell CT et al. KiSS1 suppresses metastasis in human ovarian cancer via inhibition of protein kinase C alpha. Clin Exp Metastasis 2005; 22: 369-376.

19 Nash KT, Welch DR. The KISS1 metastasis suppressor: mechanistic insights and clinical utility. Front Biosci 2006; 11: 647-659.

20 Ohtaki T, Shintani Y, Honda S, Matsumoto H, Hori A, Kanehashi $\mathrm{K}$ et al. Metastasis suppressor gene KiSS-1 encodes peptide ligand of a G-protein-coupled receptor. Nature 2001; 411: 613-617.

21 Nash KT, Phadke PA, Navenot JM, Hurst DR, AccavittiLoper MA, Sztul E et al. Requirement of KISS1 secretion for multiple organ metastasis suppression and maintenance of tumor dormancy. J Natl Cancer Inst 2007; 99: 309-321.

22 De Waard-Siebinga I, Blom DJ, Griffioen M, Schrier PI, Hoogendoorn E, Beverstock G et al. Establishment and characterization of an uveal-melanoma cell line. Int J Cancer 1995; 62: 155-161.

23 Diebold Y, Blanco G, Saornil MA, Fernandez N, Lazaro MC. Morphologic and immunocytochemical characterization of four human uveal cell lines (melanoma- and melanocytesderived). Curr Eye Res 1997; 16: 487-495.

24 Steeg PS. Metastasis suppressors alter the signal transduction of cancer cells. Nat Rev Cancer 2003; 3: 55-63.

25 Ueno H, Nakamura H, Inoue M, Imai K, Noguchi M, Sato H et al. Expression and tissue localization of membrane-types 1,2 , and 3 matrix metalloproteinases in human invasive breast carcinomas. Cancer Res 1997; 57: 2055-2060.

26 Nomura H, Sato H, Seiki M, Mai M, Okada Y. Expression of membrane-type matrix metalloproteinase in human gastric carcinomas. Cancer Res 1995; 55: 3263-3266.

27 El-Shabrawi Y, Ardjomand N, Radner H, Ardjomand N. MMP-9 is predominantly expressed in epithelioid and not spindle cell uveal melanoma. J Pathol 2001; 194: 201-206.

28 Yan C, Wang H, Boyd DD. KiSS-1 represses 92-kDa type IV collagenase expression by down-regulating NF-kappa B binding to the promoter as a consequence of Ikappa Balpha -induced block of p65/p50 nuclear translocation. J Biol Chem 2001; 276: 1164-1172.

29 Takino T, Koshikawa N, Miyamori H, Tanaka M, Sasaki T, Okada $\mathrm{Y}$ et al. Cleavage of metastasis suppressor gene product KiSS-1 protein/metastin by matrix metalloproteinases. Oncogene 2003; 22: 4617-4626. 